\title{
Distribution of CD31 on CD4 T-Cells from Cord Blood, Peripheral Blood and Tonsil at Different Stages of Differentiation
}

\author{
Raul Ruiz-Hernandez ${ }^{1}$, Antoni Jou ${ }^{2}$, Cecilia Cabrera ${ }^{1}$, Ferdinand Noukwe ${ }^{1}$, Josep deHaro ${ }^{3}$, \\ Francesc Borras ${ }^{4}$, Julia Blanco ${ }^{1}$, Marco Fernandez ${ }^{5}$, Bonaventura Clotet ${ }^{1}$, Lidia Ruiz ${ }^{1}$ and \\ Margarita Bofill ${ }^{*}, 1,6$
}

\author{
${ }^{I}$ Fundació irsiCaixa-HIVACAT, Badalona, Spain \\ ${ }^{2}$ Fundació Lluita contra la Sida, Hospital Universitari Germans Trias i Pujol, Badalona, Spain \\ ${ }^{3}$ Otorhinolaryngology Unit, Hospital Municipal, Badalona, Spain \\ ${ }^{4}$ Laboratori d'Immunobiologia per a la Recerca i Aplicacions Diagnòstiques, Centre de Transfusió i Banc de Teixits, \\ Fundació Germans Trias i Pujol, Badalona, Spain \\ ${ }^{5}$ Cytometry Unit, Institut d'Investigació en Ciències de la Salut Germans Trias i Pujol, Badalona, Spain \\ ${ }^{6}$ Institució Catalana de Recerca i Estudis Avançats, Barcelona, Spain
}

\begin{abstract}
CD31+ is a marker for recent thymic emigrants. Nevertheless it is present in a proportion of memory cells. We looked at the distribution of CD31 on CD4 T-cell subpopulations. In cord blood, CD31 was present in the majority of the $\mathrm{CD}^{2} 5 \mathrm{RA} \mathrm{A}^{\text {high }}$ and $60 \%$ of the CD45RA ${ }^{\text {low }}$ cells, and in adults over $70 \%$ of "true" naïve and in $5-10 \%$ of all memory subpopulations (central memory, effector memory, follicular helper and $\mathrm{T}$ regulatory cells). No major differences were seen in the distribution of chemokine receptors between $\mathrm{CD} 31+$ and $\mathrm{CD} 31$ - populations within the naïve cells nor the memory populations except for CCR3 and CCR9, which were preferentially expressed in the CD31+ memory cells. The CD31 distribution and cytokine receptors was similar between HIV negative and positive individuals, and between adult blood and tonsils. There was a correlation between the levels of TRECs and the percentages of CD31 in all samples studied.
\end{abstract}

Keywords: HIV, CD31, recent thymic emmigrants, thymus, TRECS, chemokine receptors, cord blood, tonsil.

\section{INTRODUCTION}

CD31, also known as the Platelet cell adhesion molecule 1 (PECAM 1), is a surface glycoprotein of $130 \mathrm{kDa}$ that belongs to the immunoglobulin super family [1]. This molecule is involved in the migration of cells through the endothelium cell junction and mediates cell-to-cell adhesion [2]. CD31 is expressed on a diversity of cell types including thymocytes, lymphocytes, endothelial cells, circulating monocytes, and granulocytes [1].

Because CD31 is expressed in the majority of thymic cells and on $85 \%$ of CD4 cells from cord blood $[3,4]$ it has been suggested that CD31 is a useful marker for recent thymic emigrants. Besides, the CD31+ populations in adults contain very high levels of T-Cell Receptor Excision Circles (TRECs) an episomal DNA produced during the rearrangement of the TCR in the thymus (where the level of TRECs is very high). The episomal DNA is diluted during T cell division and accordingly, naïve $\mathrm{T}$ cells have higher levels of TRECs that memory cells. In adults, CD31 is expressed in CD45RA+ subpopulation of CD4+T-cells that contains the majority of TRECs detected on CD4 T-cells [4].

*Address correspondence to this author at the Fundació irsiCaixa, Ctra. de Canyet s/n, 08916, Badalona, Spain; Tel: 3493465 6374; Fax: 3493 4653968; E-mail: mbofill@irsicaixa.es
This population has been named as ${ }^{\text {Thymic }}$ naïve CD4 $\mathrm{T}$ cells [5], a cell population that expands in the periphery under homeostatic stimuli. The CD45RA+ CD31 - cell population has been called ${ }^{\text {central }}$ naïve and it expands through TcR engagement [5]. CD31 is also expressed on about $10 \%$ of CD4 memory Tcells $[3,4]$, and accordingly, small amounts of TRECs have also been observed in the memory population.

The expression of CD45RA and CD45RO, two isoforms of the CD45 family, has classically been used to define naïve and memory CD4 T. Nevertheless, CD4 T cells are functionally and phenotypically heterogeneous depending on the antigenic stimuli, the environment where the cells are located, the state of differentiation of the cells, etc [6]. In this study, we have compared the distribution of CD31 on a antigen naïve and antigen primed environment by studying CD4 $\mathrm{T}$ cells from cord blood and peripheral blood from adult. Cord blood CD4 T cells do not usually express CD45RO. However, there are two CD45RA populations regarding the intensity of expression of CD45RA: a CD45RA high and a CD45RA ${ }^{\text {low }}$, the latter being virtually absent in adult PBMCs [7]. We then compared the levels of TRECs within these populations and observed that the levels of TRECs were higher in the CD45RA high than the $\mathrm{CD}_{5} \mathrm{RA}^{\text {low }} \mathrm{CD} 4 \mathrm{~T}$-cells. Second, in order to see whether the 4 major CD31+ and CD31 - naïve and memory population had different expression of chemokine receptors, we compared their distribution of CXCR3, CXCR5, CCR4, CCR5, CCR6, CCR3 
and CCR9 from peripheral blood and tonsils. Third, as the naïve and memory populations are heterogeneous we expanded these studies by looking the expression of CD31 within the following "naïve" and "memory" CD4 subpopulation: CD45RA+, CD45RO-, CCR7+ CD127+ CCR7+cells ("true" naïve populations), CD45RA-, CD45RO+ CCR7-+ (central memory), CD45RO+CCR7- CD4 T CD45RA+ (effector memory [8]), CD45RA- CD45RO+ CXCR5+ (follicular helper) [9] the CD45RA- CD45RO+ CD25 bright ( $\mathrm{T}$ regs) [10] populations, and the CD45RA+ CD45RO+, CD27-, CCR7- and CD62Lreverted memory population.

Finally because HIV infection can have a dramatic effect on CD4 T cells [11] we compared the correlation of TRECs and CD31 and the expression of chemokine receptors between healthy donors and HIV+ patients to check how $\mathrm{HIV}$ could affect $\mathrm{CD} 4{ }^{\text {thymic }}$ naïve populations.

\section{MATERIALS AND METHODOLOGY}

\section{Subjects and Samples}

Blood samples from healthy adult volunteers, buffy coats and cord blood were provided by the Centre de Transfusions i Banc de Teixits (Valle Hebron Hospital, Barcelona, Spain). Peripheral blood from $34 \mathrm{HIV}$-infected volunteers that attended the VIH clinic was provided by the Hospital Universitari Germans Trias I Pujol. Twelve out of the 34 patients had never received antiretroviral therapy. Tonsils (n $=15$ ) and peripheral blood were obtained from patients who underwent routine tonsillectomy at the Hospital Municipal de Badalona (Spain). The procedures followed in the study in accordance with the Helsinki Declaration in 1975, as revised in 1983, were approved by the local Ethical Committee and all patients gave informed consent.

The tonsils were disaggregated with forceps and the tonsillar cells were stained by immunofluorescence.

\section{Isolation of CD4 T Subsets}

PBMCs from buffy coats, peripheral blood, and tonsil suspensions were isolated by density-gradient centrifugation on Ficoll-Paque gradient (Atom Reactiva, Barcelona, Spain), and resuspended in culture medium.

Isolated CD4/CD45RA+ or CD45RO+ cells were obtained by negative selection by magnetic purification (StemSep $^{\mathrm{TM}}$, StemCell Technologies Inc, Vancouver, Canada) with a purity greater than $95 \%$ and further enriched for CD31+ (90- $99 \%)$ and CD31- (1- $10 \%)$ with magnetic beads (EasySep ${ }^{\mathrm{TM}}$, StemCell Technologies Inc, Vancouver, Canada). Alternatively, T CD4+ lymphocytes were isolated by immunomagnetic separation columns (StemSep) (purity greater 95\%) and subsequently stained with CD45RA and CD31 immnunofluorescent antibodies and sorted with purity $>98 \%$ in both CD31+ and CD31- fractions.

\section{Viral Load}

Plasma HIV-1 RNA was measured in $0.5 \mathrm{~mL}$ of plasma using the Amplicor HIV-1 Monitor ultrasensitive test (Roche Diagnostics, Barcelona, Spain) a reverse transcription-PCRbased assay with a limit of detection of 50 HIV-1 RNA copies per milliliter.

\section{Flow Cytometry Analysis}

Phenotypic analysis was performed on whole blood or tonsillar cells by direct immunofluorescence using four fluorochrome combinations of reagents and monoclonal antibodies to CD4, CD45RA, CD45RO, CD62L, CD27, CD31 (Becton Dickinson, Pharmingen, Madrid, Spain), CD127, CD25, and CD69-FITC (DakoCytomation, Barcelona, Spain). Staining of CCR7 was carried out by a primary IgM mouse anti-human CCR7 antibody, isotype $\operatorname{IgM}$ (BD Pharmingen), followed by a secondary goat antibody, anti-mouse IgM FITC (BD Pharmingen). The isotype antibodies were used as negative controls. Stained samples were acquired using a FACScalibur flow cytometer (BD).

\section{Quantification of T-Cell Receptor Excision Cycles}

Genomic DNA was extracted from pellets of PBMCs tonsillar cells and isolated subsets using QIAamp DNA Blood Mini or Micro Kit (QIAgen, Valencia, CA) according to the manufacturer's instructions. 2.5.

Quantification of TREC and CCR5 was carried out by Real-Time quantitative PCR, performed in a spectrofluorometric thermal cycler (ABI PRISM 7000; Applied Biosystems, Foster City, CA). CCR5 has been used as a reference gene [12] as it is known that it only has two copies of CCR5 per cell [13]. The thermal cycling conditions were $2 \mathrm{~min}$ at $50^{\circ} \mathrm{C}, 10 \mathrm{~min}$ at $95^{\circ} \mathrm{C}$, and 50 cycles each at $95^{\circ} \mathrm{C}$ for $15 \mathrm{sec}$ and $60^{\circ} \mathrm{C}$ for $1 \mathrm{~min}$. The PCR primer and probe sequences were: TREC: forward 5'-CACATCCCTT TCAA CCATGCT- 3' and reverse 5'-GCCAGCTGCAGG GTTTA GG-3' and the fluorogenic probe was FAM - 5' -AC ACCT CTGGTTTTTGTAAAGGTGCCCACT-3' - TAMRA. CCR5 coding sequence was used to measure cell equivalents in the input DNA. CCR5 primers were: forward 5'-TCATTACAC CTGCAGCTCTCATTT- 3' and reverse 5'-ACACCGAAG CAGAGTTTTTAGGAT- 3', and the fluorogenic probe was VIC -5'-CTGGTCCTGCCGCTGCT TGTCA-3' -TAMRA. (Applied Biosystem, Warrington, Cheshire, UK).

Each $50 \mu \mathrm{l}$ reaction mixture contained $25 \mu \mathrm{l}$ Taqman Universal PCR Master Mix (PE Applied Biosystems, Foster City, CA), or $250 \mathrm{nM}$ of each TREC primer and $160 \mathrm{nM}$ TREC probe or $300 \mathrm{nM}$ CCR5 forward primer, $900 \mathrm{nM}$ CCR5 reverse primer and $150 \mathrm{nM}$ CCR5 probe and $5 \mu 1$ of DNA sample.

Standard serial dilutions (from 40000 to 10 copies for TRECs, and $10^{6}$ to $10^{2}$ copies for CCR5) of plasmid DNA containing the signal-joint TREC or CCR5 fragment in the pGEM T Easy Vector (Promega, Barcelona, Spain) were used to quantify TRECs and the number of cells in each sample. Samples were analysed in triplicate. Values with a deviation higher than $0.3 \mathrm{Ct}$ were discarded and the results obtained were averaged. Results were expressed as TREC copies/million cells (2 X CCR5 copies) [12, 13].

\section{Statistical Analysis}

Statistical analysis was performed with GraphPad Prism 4.0 software (GraphPad Software Inc, San Diego, CA). When comparing two groups we used the Mann-Whitney test followed by the Wilcoxon test if the results were obtained from the same samples. Non-parametric distribution was assumed and significance was set at $\mathrm{p}<0.01$. 


\section{RESULTS AND DISCUSSION}

\section{Expression of CD31 and TRECs on CD4 T-Cells from Cord Blood}

In order to study the expression of CD31 in an antigenfree environment, we analysed the expression of CD31 on CD4 T-cell subsets from cord blood, where the fetal immune system is unlikely to have been in contact with foreign antigens. Accordingly, the majority of cord blood CD4 Tcells are CD45RO- [7], the latter being a marker that is used to detect memory cells in adults (Fig. 1). In contrast, the expression of CD45RA was heterogeneous (Fig. 1a), with cells that expressed high to negligible levels of CD45RA. Over $85 \%$ of CD45RA ${ }^{\text {high }}$ cells expressed CD31 while the CD45RA ${ }^{\text {low }}$ population contained only $60 \%$ of cells positive for CD31. Furthermore, the CD45RA ${ }^{\text {high }}$ population contained two to three times higher levels of TRECs than the CD45RA ${ }^{\text {low }}\left(27.5 \pm 5.1\right.$ versus $13.3 \pm 4.05$ copies $\times 10^{6}$ cells; $(\mathrm{p}=0.02)($ Fig. 2$)$ suggesting a possible pathway of differentiation from thymic medullary $\mathrm{T}$-cells to recent thymic emigrants $\left(\mathrm{CD}_{4} 5 \mathrm{RA}^{\text {high }} / \mathrm{CD} 31+\right)$, to a CD45RA ${ }^{\text {low }}$ $\mathrm{CD} 31+$ and a CD45RA ${ }^{\text {low }} \mathrm{CD} 31-$ population in cord blood. In adults, and after antigenic stimulation, this population might give rise to two adult populations: $\mathrm{CD}^{2} 5 \mathrm{RO}^{\text {high }}$, CD45RA low. CD31+ and CD45RO ${ }^{\text {high }}$, CD45RA ${ }^{\text {low }} \mathrm{CD} 31-$.' This hypothesis is supported by the fact that after TcR activation in vitro the CD4+CD45RA ${ }^{\text {high }}$. T-cells down- regulate the level of CD45RA and up-regulate CD45RO, indicating that antigenic challenge might be responsible for the induction of CD45RO in adults [14]. From that point of view it is possible that CD45RA ${ }^{\text {low }}$ cells in cord blood are the equivalent of memory cells from antigen-exposed individuals.

\section{Distribution of CD31+ Cells in the CD4 Populations from Peripheral Blood and Tonsils from Adults}

Naive and memory cells have different re-circulation patterns: while naïve T-cells traffic from the peripheral blood to the lymph nodes through the high endothelium venules, memory T-cells travel from the peripheral tissue to the lymph nodes via the afferent lymph [15]. As CD31 is involved in the transmigration of lymphoid cells through high endothelial venules, we compared the populations from peripheral blood and tonsils obtained from the same subjects in order to see whether any of the populations were selectively retained in the tissues. Furthermore we compared the chemokine receptors profiles on these populations.

As shown in Table 1, the homeostatic receptors CCR7 and CXCR4 were present in both positive and negative CD31 naïve memory subsets. The inflammatory chemokine receptors (CXCR3, CXCR5, CCR4, CCR5, CCR6, CCR3 and CCR9) were restricted to the two positive and negative memory CD31 populations. We did not find any major
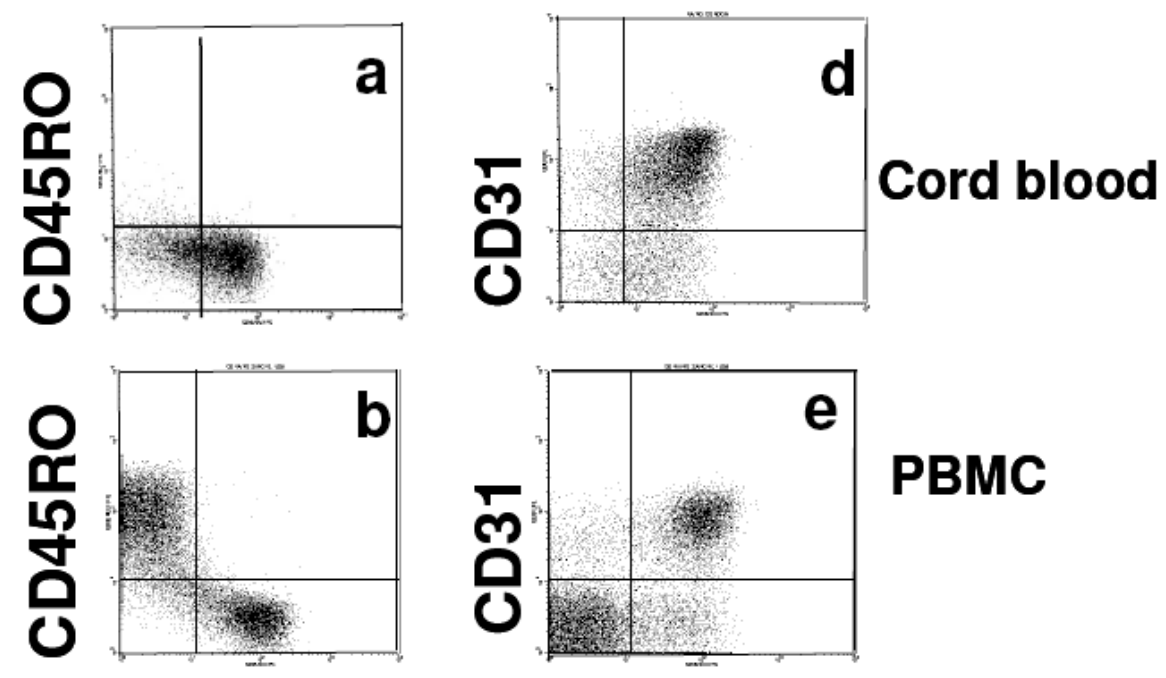

\section{PBMC}
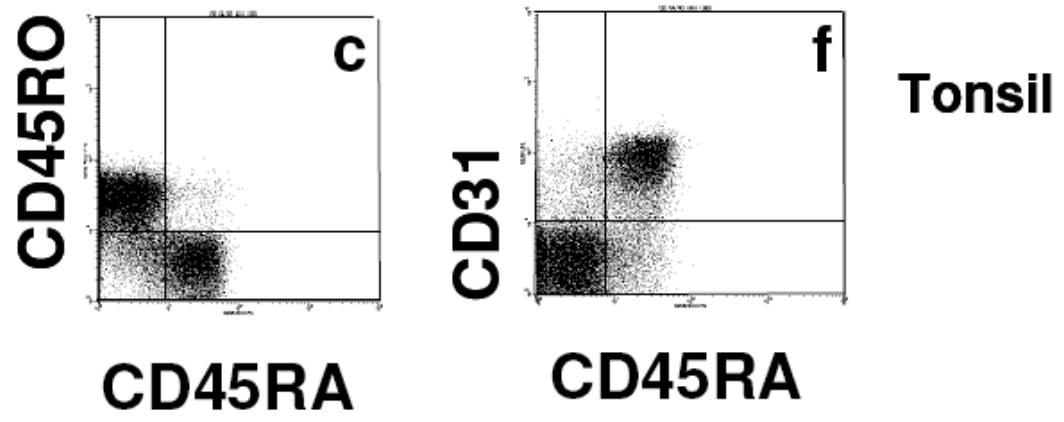

Fig. (1). Percentage of CD31 + CD4 T-cells and levels of TRECs in the different CD4 T-cell populations from cord blood. Mononuclear cells from cord blood, peripheral blood and tonsil were stained with antibodies to CD4, CD45RA, CD45RO and CD31. The figure shows the expression of CD45RA and CD45RO (a-c) or CD45RA and CD31 (d-f) on CD4-gated T-cells in one representative experiment. Fig. (a) shows a very low expression of CD45RO in cord blood and the presence of a CD45RA ${ }^{\text {low }} \mathrm{CD}^{4} \mathrm{RO}^{\text {low }}$ population present in cord blood but in very in low numbers in adults. 
Table 1. Expression of the Percentage CD31+ Cells in Naïve and Memory CD4 T-Cells in Adults

\begin{tabular}{|c|c|c|c|c|}
\hline & & PBMC (HIV neg) N = 14 & $\operatorname{PBMC}(\mathrm{HIV}+) \mathrm{N}=12$ & Tonsil $N=11$ \\
\hline NAIVE & CD45RA+RO- & $72.4 \pm 2.3$ & $77.37 \pm 2.9$ & $87.5 \pm 1.9$ \\
\hline \multirow[t]{4}{*}{ True naive } & CD45RA+CCR7+ & $72.7 \pm 4.4$ & $76.9 \pm 2.9$ & $83.5 \pm 1.7$ \\
\hline & CD45RA+CD62L + & $73.6 \pm 4.7$ & $76.9 \pm 2.9$ & $88.8 \pm 1.4$ \\
\hline & CD45RA+CD27+ & $72.9 \pm 4.6$ & $78.4 \pm 2.8$ & $84.6 \pm 1.5$ \\
\hline & CD45RA+CD127+ & $71.4 \pm 5.3$ & $74.9 \pm 2.9$ & $81.9 \pm 1.8$ \\
\hline Naive $T$ regs & CD45RA+CD127- & $56.2 \pm 6.7$ & $51.2 \pm 5.6$ & $73.4 \pm 2.2$ \\
\hline MEMORY & CD45RO+RA- & $6.6 \pm 0.4$ & $7.2 \pm 0.4$ & $11.4 \pm 0.7$ \\
\hline Central memory & CD45RA-CCR7+ & $12.1 \pm 1.6$ & $17.5 \pm 1.7$ & $4.3 \pm 1.6$ \\
\hline Effector memory & CD45RA-CCR7- & $4.7 \pm 0.4$ & $6.4 \pm 1.4$ & $4.1 \pm 0.7$ \\
\hline Follicular helper & CD45RA-CXCR5 & $7.0 \pm 0.8$ & $5.9 \pm 1.8$ & $4.9 \pm 0.3$ \\
\hline T regulatory & CD45RA-CD127- & $6.8 \pm 1.0$ & $7.8 \pm 1.6$ & $6.5 \pm 0.9$ \\
\hline \multicolumn{5}{|c|}{ REVERTED MEMORY } \\
\hline & CD45RA+CCR7- & $35.4 \pm 7.3$ & $21.5 \pm 6.6$ & $84.0 \pm 2.8$ \\
\hline & CD45RA+CD62L- & $29.3 \pm 5.2$ & $15.8 \pm 3.8$ & $71.3 \pm 3.4$ \\
\hline & CD45RA+CD27- & $12.3 \pm 3.5$ & $7.7 \pm 1.6$ & $85.5 \pm 2.5$ \\
\hline
\end{tabular}

The data is expressed as Mean and Standard Error of Mean. PBMC = peripheral blood mononuclear cells.

differences between the percentages of these cytokine receptors between CD31 positive and CD31 negative cells from peripheral blood or tonsillar cells, except for the presence of CCR3 and CCR9, which were more numerous in the CD31+ than in the CD31- cells. The ligands for these two receptors attract cells to the mucosal sites [16, 17]. These populations were nevertheless absent from tonsillar cells, indicating that the cells in mucosal tissues might have a different circulation pathway compared to inflammatory cells.

We then compared the presence of TRECs in the PBMCs and tonsillar cells to elucidate the rate of expansion of the naïve cells at both sites. As mentioned above, the loss of CD45RA was closely mirrored by a decrease in the percentage of CD31+ cells. The majority of TRECs were found in the CD45RA+ CD31+ cells compared to the CD45RA + CD31- cells and memory populations, and the levels of TRECs present in these populations in blood and tonsils were very similar except for the CD45RA+ CD31population, in which the levels of TRECs in the tonsils were twice as high as that in peripheral blood (Fig. 2b).

We then looked at the distribution of CD31 in the CD4 T-cell subpopulations shown in Table 1. The percentage of CD31 on true naïve CD4 T-cells was identified by the coexpression of CD45RA and CCR7, CD127, CD62L or CD27. Over $90 \%$ of CD45RA+ CD4 T-cells expressed CCR7, CD62L, CD27 and CD127. CD31 was present in around $70 \%$ of the cells in these populations (Table 1) from both peripheral blood and tonsils.

It has been postulated that a small proportion of the CD45RA+ CD127- population can be identified as a precursor of a subtype of regulatory T-cells [18]. We found that $56.2 \pm 6.7 \%$ of adult peripheral blood and $73.4 \pm 12.2 \%$ of tonsillar cells with this phenotype also expressed CD31. These values are intermediate between those observed in the CD45RA+ naïve and CD45RA- memory populations, indicating that CD31 might be lost during differentiation towards regulatory T-cells.

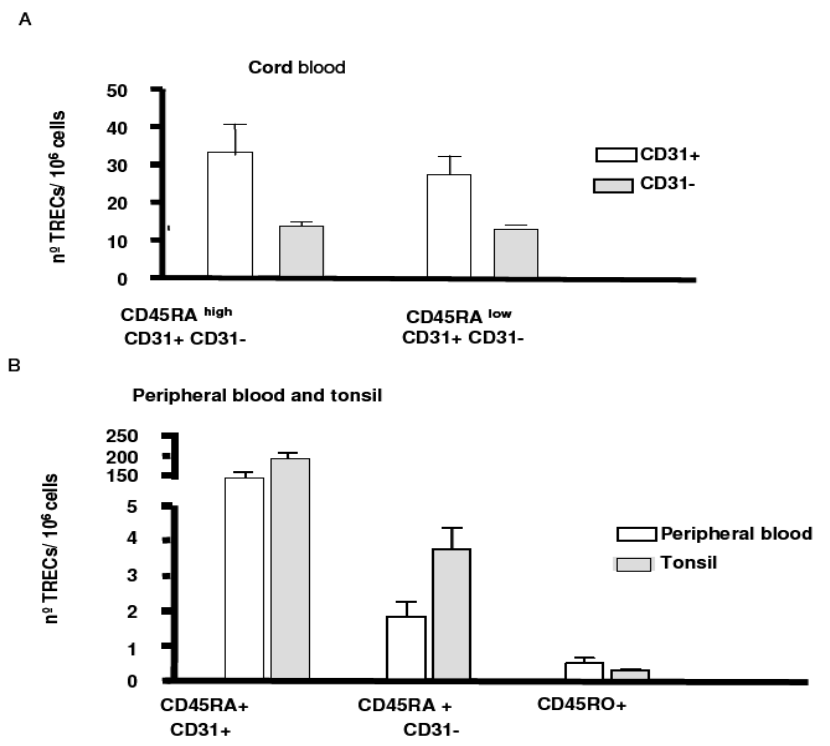

Fig. (2). Percentage of CD31 + CD4 T-cells and levels of TREC in CD4 subsets from cord blood, peripheral blood and tonsil samples. A) Cord blood CD4 CD45RA ${ }^{\text {high }}$ and CD45RA ${ }^{\text {low }}$ CD4 Tcells were isolated magnetic beads, and further sorted by the expression of CD31. The levels of T-cell receptor excision circles within each fraction were measured as described in the Material and methods. The bars show the number of TRECs $\times 10^{6}$ cells in the CD45RA ${ }^{\text {high. }}$ CD31 positive and neg populations and in the $\mathrm{CD}_{45 \mathrm{RA}^{\text {low }}} \mathrm{CD} 31+$ and $\mathrm{CD} 31$ neg populations The data is expressed as mean \pm SEM. B) The CD45RA+ CD31+, CD45RA+ CD31 negative and CD45RO+ CD4 T-cells from peripheral blood (white bars) and tonsils (grey bars) were isolated as described in material and methods. The levels of T-cell receptor excision circles (TRECs) were measured as described in the Material and methods. The bars show the number of TRECs x $10^{6}$ cells within the different subpopulations. The data is expressed as mean $\pm \operatorname{SEM}(n=4)$. 
Table 2. Percentage of Cells Expressing Chemokine Receptors in CD4 Naïve and Memory CD31+ and CD31-Populations

\begin{tabular}{|c|c|c|c|c|c|}
\hline & & \multicolumn{2}{|c|}{ CD45RA+ } & \multicolumn{2}{|c|}{ CD45RA neg } \\
\hline & & CD31+ & CD31- & CD31+ & CD31- \\
\hline \multicolumn{6}{|c|}{ Homeostatic Chemokine Receptors } \\
\hline \multirow[t]{3}{*}{ CCR7 } & HIV neg & $96.4 \pm 2.8$ & $85.1 \pm 14.1$ & $71.9 \pm 13$ & $53.1 \pm 10.7$ \\
\hline & HIV pos & $97.2 \pm 1.51$ & $84.3 \pm 12.0$ & $68.7 \pm 4.65$ & $50.85 \pm 6.77$ \\
\hline & Tonsil & $78.9 \pm 1.6$ & $71.72 \pm 18.6$ & $67.2 \pm 16.6$ & $46.5 \pm 27.71$ \\
\hline \multirow[t]{3}{*}{ CXCR4 } & HIV neg & $78.4 \pm 13.7$ & $62.2 \pm 17.84$ & $56.8 \pm 16.9$ & $34.3 \pm 16.5$ \\
\hline & HIV pos & $90.1 \pm 7.62$ & $78.8 \pm 9.93$ & $70.6 \pm 23.1$ & $55.8 \pm 20.5$ \\
\hline & Tonsil & $72.1 \pm 29.4$ & $67.7 \pm 28.5$ & $58.6 \pm 26.7$ & $57.6 \pm 29.3$ \\
\hline \multicolumn{6}{|c|}{ Inflammatory Chemokine Receptors } \\
\hline \multirow[t]{3}{*}{ CXCR3 } & HIV neg & $4.5 \pm 3.05$ & $9.8 \pm 6.44$ & $25.0 \pm 10.5$ & $37.4 \pm 8.85$ \\
\hline & HIV pos & $1.68 \pm 1.24$ & $8.1 \pm 8.49$ & $22.2 \pm 18.2$ & $32.6 \pm 14.5$ \\
\hline & Tonsil & $2.71 \pm 2.71$ & $6.7 \pm 3.96$ & $16.2 \pm 3.16$ & $11.2 \pm 2.45$ \\
\hline \multirow[t]{3}{*}{ CXCR5 } & HIV neg & $2.4 \pm 1.55$ & $4.5 \pm 2.86$ & $35.4 \pm 7.3$ & $21.5 \pm 6.6$ \\
\hline & HIV pos & $2.1 \pm 2.35$ & $2.35 \pm 1.29$ & $29.3 \pm 5.2$ & $15.8 \pm 3.8$ \\
\hline & Tonsil & $5.0 \pm 3.5$ & $5.8 \pm 5.14$ & $6.46 \pm 3.31$ & $14.2 \pm 5.39$ \\
\hline \multirow[t]{3}{*}{ CCR4 } & HIV neg & $3.8 \pm 2.44$ & $4.0 \pm 1.34$ & $51.8 \pm 10.4$ & $52.1 \pm 8.95$ \\
\hline & HIV pos & $2.6 \pm 1.67$ & $3.7 \pm 1.85$ & $38.8 \pm 15.4$ & $52.3 \pm 13.0$ \\
\hline & Tonsil & $7.0 \pm 4.5$ & $10.2 \pm 7.78$ & $15.7 \pm 2.75$ & $26.9 \pm 11.5$ \\
\hline \multirow[t]{3}{*}{ CCR5 } & HIV neg & $3.2 \pm 3.4$ & $7.1 \pm 8.65$ & $29.5 \pm 18.7$ & $21.0 \pm 7.94$ \\
\hline & HIV pos & $0.74 \pm 0.33$ & $3.68 \pm 2.5$ & $13.4 \pm 8.77$ & $19.9 \pm 9.17$ \\
\hline & Tonsil & $2.26 \pm 1.03$ & $4.03 \pm 2.33$ & $20.7 \pm 8.21$ & $9.7 \pm 2.14$ \\
\hline \multirow[t]{3}{*}{ CCR6 } & HIV neg & $1.76 \pm 1.17$ & $1.1 \pm 1.02$ & $17.9 \pm 15.1$ & $22 \pm 13.3$ \\
\hline & HIV pos & $0.87 \pm 0.9$ & $1.7 \pm 2.38$ & $7.6 \pm 3.15$ & $24.4 \pm 6.63$ \\
\hline & Tonsil & $8.4 \pm 3.96$ & $5.7 \pm 2.01$ & $3.9 \pm 1.64$ & $2.9 \pm 2.5$ \\
\hline \multirow[t]{3}{*}{ CCR3 } & HIV neg & $3.02 \pm 4.51$ & $1.5 \pm 1.91$ & $10.4 \pm 9.69$ & $1.84 \pm 1.59$ \\
\hline & HIV pos & $0.7 \pm 0.18$ & $0.54 \pm 0.23$ & $5.9 \pm 3.67$ & $0.85 \pm 0.66$ \\
\hline & Tonsil & $2.53 \pm 1.33$ & $1.82 \pm 1.1$ & $4.2 \pm 2.03$ & $1.7 \pm 0.80$ \\
\hline \multirow[t]{3}{*}{ CCR9 } & HIV neg & $4.86 \pm 5.23$ & $1.85 \pm 1.36$ & $20.2 \pm 17.6$ & $2.4 \pm 1.54$ \\
\hline & HIV pos & $7.37 \pm 7.78$ & $5.92 \pm 6.74$ & $15.6 \pm 13.7$ & $7.2 \pm 6.63$ \\
\hline & Tonsil & $4.29 \pm 1.66$ & $3.07 \pm 1.11$ & $6.5 \pm 1.83$ & $2.4 \pm 1.57$ \\
\hline
\end{tabular}

The data shows the percentage of the different chemokine receptors and it is expressed as mean \pm Standard Error of Mean.
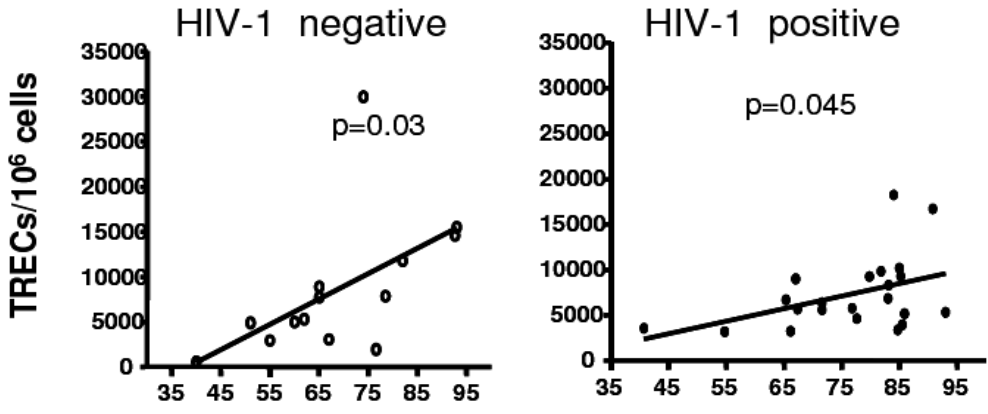

$\%$ of CD31+ cells in the CD4+ CD45RA+ population

Fig. (3). Correlation between the number of TRECs and percentage of CD31+ cells in the CD4+ CD45RA+ population in HIV negative and HIV+ subjects. The CD4 + CD45RA $+\mathrm{T}$ cells from peripheral blood were isolated by magnetic beads from a cohort of HIV negative $(n=14)$ and HIV infected $(n=22)$ subjects. The levels of T-cell receptor excision circles within each fraction were measured as described in the material and methods and the percentage of CD31+ cells in the fractionated population was measured by flowcytometry. The figure shows a positive and statistically significant correlation between the number of TRECs and the percentage of CD31 positive cells in both HIV negative and HIV positive subjects. 
Table 3. Patients Characteristics

\begin{tabular}{|c|c|c|c|c|c|c|}
\hline 1 & 38 & 401 & $<50$ & 15 & 7 & 5 \\
\hline 2 & 56 & 377 & $<50$ & 8 & 7 & 6 \\
\hline 3 & 32 & 264 & $<50$ & 13 & 8 & 2 \\
\hline 6 & 31 & 194 & $<50$ & 2 & 2 & 1 \\
\hline 7 & 41 & 322 & $<50$ & 9 & 8 & 4 \\
\hline 8 & 55 & 446 & $<50$ & 16 & 9 & 0.6 \\
\hline 9 & 67 & 572 & $<50$ & 16 & 11 & 6 \\
\hline 12 & 31 & 510 & $<50$ & 4 & 4 & 3 \\
\hline 13 & 51 & 449 & $<50$ & 9 & 8 & 5 \\
\hline 14 & 46 & 469 & $<50$ & 11 & 10 & 0.5 \\
\hline 15 & 58 & 575 & $<50$ & 18 & 11 & 2 \\
\hline 16 & 44 & 606 & $<50$ & 9 & 8 & 1 \\
\hline 17 & 47 & 162 & $<50$ & 9 & 9 & 7 \\
\hline 18 & 58 & 707 & $<50$ & 11 & 10 & 7 \\
\hline 19 & 41 & 932 & $<50$ & 17 & 3 & 3 \\
\hline 26 & 28 & 355 & 30000 & 2 & Naive & NA \\
\hline 27 & 25 & 1236 & 33000 & 1 & Naive & NA \\
\hline 28 & 29 & 824 & 6200 & 4 & Naive & NA \\
\hline 29 & 46 & 748 & 15000 & 1 & Naive & NA \\
\hline 30 & 36 & 465 & 79000 & 2 & Naive & NA \\
\hline 31 & 40 & 87 & $<50$ & 1 & Naive & NA \\
\hline 32 & 43 & 717 & $<50$ & 1 & Naive & NA \\
\hline 33 & 29 & 377 & 11000 & 1 & Naive & NA \\
\hline 34 & 26 & 638 & 21000 & 1 & Naive & NA \\
\hline
\end{tabular}

CD4 expressed as CD4 cells per cubic millimetre.

VL = Viral Load expressed in HIV-1 RNA copies/millilitre (Amplicor HIV-1 Monitor ultrasensitive test, (Roche, Diagnostics, Barcelona, Spain).

$\mathrm{NA}=$ non applicable.

Naïve $=$ Subjects that had not received antiretroviral therapy.

We then focussed our attention on the subpopulations of memory CD45RO+ cells (Table 1). Only 6 to $10 \%$ of the $\mathrm{CD} 45 \mathrm{RO}+$ cells were $\mathrm{CD} 31+$, but this small percentage of positive cells was also present in all subsets studied, including the follicular helper population (CD45RO+ CXCR5 +) and the regulatory T-cells (CD45RO+ CD127-). There was nevertheless a small but significant difference in the percentage of CD31+ cells between the CCR7- central 
and CCR7+ effector memory populations $(12.1 \pm 1.6 v s 4.7$ $\pm 0.4 \%, \mathrm{p}=0.0001)$. This difference was not observed in tonsils, where the percentage of CD31 cells was similar to that of peripheral blood effector memory cells $(4.7 \pm 0.4 v s$ $4.1 \pm 0.7)$.

In sharp contrast, the percentage of CD31+ cells in the peripheral blood reverted memory populations, defined by the expression of CD45RA and lack of expression of CCR7, CD62L or CD27, was very heterogeneous $(35.4 \pm 7.3 \%$; 29.3 $\pm 5.2 \%$ and $12.3 \pm 3.5 \%$ respectively) and involved percentages of $\mathrm{CD} 31+$ cells that were intermediate between memory and naïve CD4+ T-cells. This suggests that the subpopulations identified by the lack of expression of CCR7, CD62L or CD27 were not identical and that more specific markers are needed to identify this population. In contrast, the percentage of $\mathrm{CD} 31$ in the corresponding tonsillar populations (CD45RO+, CCR7-, CD62L- and CD27-) was similar to the levels observed in naïve cells $(84.0 \pm 2.8 \%$; $71.3 \pm 3.4 \%$ and $85.5 \pm 2.5 \%$, respectively).

\section{HIV Infection}

Because HIV infection can damage both thymic and peripheral CD4 T-cells [11], we were interested to know whether HIV infection could influence the expression of CD31. To answer this question we analysed 34 HIV infected subjects from which 12 had not previously received antiretroviral therapy (Table 3). We first looked at whether CD31 also correlated with the amount of TRECs present in the samples (Fig. 3), and indeed, we found a positive and significant correlation between the percentage of CD31+ cells and the number of TRECs present in the populations isolated from HIV negative and positive individuals (Fig. 3) (HIV negative $\mathrm{N}=: \mathrm{r}=0.3$ and HIV positive: $\mathrm{r}=0.2$; Subjects 1 to 22). As in HIV non-infected subjects, the majority of TRECs were present in the CD4+ CD45RA+ CD31+ cells (data not shown).

Furthermore, we found no statistically significant differences between the percentage of CD31+ cells neither within the CD4 subpopulations (Table 1) nor in the expression of chemokine receptors (Table 2) in HIV negative and HIV positive patients who had not received antiretroviral therapy (Patients 23 to 34 from Table 1). Therefore, CD31 can be used as a marker for recent thymic emigrants in HIVinfected patients. The use of CD31as a marker for recent thymic emigrants has an advantage on the use of the number of TRECs in that the measurement of CD31 is much simpler, quicker and can be carried out at a single cell level in whole blood without the need to isolate the relevant populations.

In summary, we have described a population of cells in cord blood that may be the equivalent of memory cells in an antigen naïve environment. These cells acquire CD45RO and lose CD45RA upon antigenic stimulation in vitro.

We have shown the distribution of CD31 among naïve and memory subsets and shown that all memory subpopulations have around $10 \%$ of cells that express CD31. Although CD31 is involved in the transmigration of cells we did not find any differences between peripheral blood and tonsillar cells. Finally, we have shown that CD31 also correlates with the number of TRECs in an untreated HIV population and is a marker that can be used to follow recent emigrants in clinical trials.

\section{ACKNOWLEDGEMENTS}

This work was funded in part by the Fundació irsiCaixa and Red Temática de Investigación en SIDA, (RG GO3/173), FIS 1081308 and HIVACAT.

The authors thank the patients who volunteered for this study, and the nursing staff who assisted at the site of the study.

\begin{tabular}{lll}
\multicolumn{2}{l}{ ABBREVIATIONS } \\
TRECs & $=$ & T-cell receptor excision circles \\
HIV & $=$ & Human Immunodeficiency virus \\
$\mathrm{IL}$ & $=$ & Interleukine \\
$\mathrm{T}$ reg & $=$ & T regulatory cells \\
$\mathrm{CD}$ & $=$ & Cluster of differentiation \\
PECAM-1 & $=$ & Platelet cell adhesion molecule 1 \\
Th & $=$ & T helper \\
PBMCs & $=$ & Peripheral mononuclear cells \\
MFI & $=$ & Mean fluorescence intensity
\end{tabular}

\section{REFERENCES}

[1] Newman PJ. The biology of PECAM-1. J Clin Invest 1997; 99: 38.

[2] Albelda SM, Muller WA, Buck CA, Newman PJ. Molecular and cellular properties of PECAM-1 (endoCAM/CD31): a novel vascular cell-cell adhesion molecule. J Cell Biol 1991; 114: 105968.

[3] Stockinger H, Schreiber W, Majdic O, Holter W, Maurer D, Knapp W. Phenotype of human T cells expressing CD31, a molecule of the immunoglobulin supergene family. Immunology 1992; 75: 538.

[4] Kimmig S, Przybylski GK, Schmidt CA, et al. Two subsets of naive $\mathrm{T}$ helper cells with distinct $\mathrm{T}$ cell receptor excision circle content in human adult peripheral blood. J Exp Med 2002; 195: 789-94.

[5] Kohler S, Thiel A. Life after the thymus: CD31+ and CD31human naive CD4+ T-cell subsets. Blood 2009; 113: 769-74.

[6] Bofill M, Martinez-Picado J, Ruiz-Hernandez R, et al. Naive $\mathrm{CD} 4(+) \mathrm{T}$ cells and recent thymic emigrant levels in treated individuals with HIV: clinical relevance. AIDS Res Hum Retroviruses 2006; 22: 893-6.

[7] Bofill M, Akbar AN, Salmon M, Robinson M, Burford G, Janossy G. Immature CD45RA(low)RO(low) $\mathrm{T}$ cells in the human cord blood. I. Antecedents of CD45RA+ unprimed T cells. J Immunol 1994; 152: 5613-23.

[8] Sallusto F, Kremmer E, Palermo B, et al. Switch in chemokine receptor expression upon TCR stimulation reveals novel homing potential for recently activated T cells. Eur J Immunol 1999; 29: 2037-45.

[9] Aust G, Sittig D, Becherer L, et al. The role of CXCR5 and its ligand CXCL13 in the compartmentalization of lymphocytes in thyroids affected by autoimmune thyroid diseases. Eur J Endocrinol 2004; 150: 225-1131.

[10] Taams LS, Smith J, Rustin MH, Salmon M, Poulter LW, Akbar AN. Human anergic/suppressive CD4(+)CD25(+) T cells: a highly differentiated and apoptosis-prone population. Eur $\mathrm{J}$ Immunol 2001; 31: 1122- 31.

[11] Phillips AN, Sabin CA, Elford J, Bofill M, Janossy G, Lee CA Acquired immunodeficiency syndrome (AIDS) risk in recent and long-standing human immunodeficiency virus type 1 (HIV-1)infected patients with similar CD4 lymphocyte counts. Am J Epidemiol 1993; 138: 870-8.

[12] Beloukas A, Paraskevis D, Haida C, Sypsa V, Hatzakis A. Development and assessment of a multiplex real-time PCR assay for quantification of human immunodeficiency virus type 1 DNA. J Clin Microbiol 2009; 47: 2194- 9. 
[13] Kostrikis LG, Touloumi G, Karanicolas R, et al. Quantitation of human immunodeficiency virus type 1 DNA forms with the second template switch in peripheral blood cells predicts disease progression independently of plasma RNA load. J Virol 2002; 76: 10099-108.

[14] Akbar AN, Terry L, Timms A, Beverley PC, Janossy G. Loss of CD45R and gain of UCHL1 reactivity is a feature of primed T cells. J Immunol 1988; 140: 2171-8.

[15] Mackay CR, Marston WL, Dudler L. Naive and memory T cells show distinct pathways of lymphocyte recirculation. J Exp Med 1990; 171: 801-17.
[16]

Pan J, Kunkel EJ, Gosslar U, et al. A novel chemokine ligand for CCR10 and CCR3 expressed by epithelial cells in mucosal tissues. J Immunol 2000; 65: 2943-9.

[17] Campbell JJ, Brightling CE, Symon FA, et al. Expression of chemokine receptors by lung $\mathrm{T}$ cells from normal and asthmatic subjects. J Immunol 2001; 166: 2842-8.

[18] Valmori D, Merlo A, Souleimanian NE, Hesdorffer CS, Ayyoub M. A peripheral circulating compartment of natural naive CD4 Tregs. J Clin Invest 2005; 115: 1953-62.

(C) Ruiz-Hernandez et al.; Licensee Bentham Open.

This is an open access article licensed under the terms of the Creative Commons Attribution Non-Commercial License (http://creativecommons.org/licenses/by$\mathrm{nc} / 3.0 /$ ) which permits unrestricted, non-commercial use, distribution and reproduction in any medium, provided the work is properly cited. 